\title{
Explore on Voice Parameters of Social Robots Applied in Education Industry
}

\author{
Yue Yuan ${ }^{1}$, Chih-Fu Wu ${ }^{2}$, Kai-Chieh Lin $^{3}$, Xiao Dou ${ }^{4}$ \\ ${ }^{1}$ The Graduate Institute of Design Science, Tatung University, Taipei,Taiwan \\ ${ }^{2}$ Department of Industrial Design, Tatung University, Taipei, Taiwan \\ First.yuanyueuk@126.com; Second.wcf@gm.ttu.edu.tw \\ ${ }^{3}$ Department of Industrial Design,Tatung University, Taipei, Taiwan \\ ${ }^{4}$ The Graduate Institute of Design Science, Tatung University, Taipei,Taiwan \\ Third.kclin@ttu.edu.tw ; Fourth.michelledou007@outlook.com
}

\begin{abstract}
The language communication of social robots has a great significance meaning for improving the robot's intelligence and humanity.This research explores the impact of the dialogue type and voice parameter settings of social robots in the education industry on their perceived personality traits and acceptance, and constructs the principles and models of voice applications for robots that are suitable for the education industry.The study included the influence of the educational robot's dialogue type and speech parameters, gender, fundamental frequency, and speech rate. Through the control of robot's speech and dialogue content, to measure the personality of the robot during the interaction between the subjects and the robots. The experiment invited 32 subjects to conduct VHRI robot experiments, which was found that there is a significant interactive effects among the dialogue types and voice parameters of social robots used in the education industry. In addition, the gender of sound and fundamental frequency can improve the personal characteristics of robot extroversion (E), agreeableness (A), conscientiousness $(\mathrm{C})$, openness $(\mathrm{O})$, and anti-neurotic $(\mathrm{N})$. Speech rates can improve the robot's agreeableness $(A)$, conscientiousness $(C)$, anti-neurotic $(N)$, openness $(\mathrm{O})$ and other characteristics. This study confirmed that the robot's dialogue types and voice parameter setting can be used to improve the robot's perceptual personality traits to improve its humanity. On this basis, the results will help social robots play their professional role in the application of education better and get better integrate into human society , at the same time, it provides references for the voice parameters design of social robotics in different industries in the future.
\end{abstract}

Keywords: Educational robots, Human-Robot interaction (HRI), Robot personality traits, Voice, Dialogue content

\section{Introduction}

In recent years, social robots have entered the educational environment, and predictions indicate that this trend will continue to stabilize.[1] Social robots for education include a variety of robots having different roles. Many scholars believe that robot technology provides significant new benefits in all levels of education. However, the degree of humanization of social robots is still low, and without effective emotional interaction with users, it is difficult to be as vivid and natural as human-to-human interaction, resulting in their low acceptance among the general public. By adjusting the social cues of the robots and designing effective emotional interactions to improve the humanity of the social robots, it is considered to be a key method to improve the current low acceptance status of social robots.[2]-[6]

Fong et al.[7] have done a more comprehensive robot social clue sorting, including appearance, behavior, facial expression, speech and so on. These social cues can be divided into two categories: 1.Language interaction: language content itself, sound; 2.Non-verbal interaction: appearance, action, expression, gaze.[8]Compared with the study of non-verbal cues, the study of social robotic language cues is still lacking. This may be due to the slow development of the previous artificially synthesized speech technology without major technological breakthroughs. In recent years, the development of synthetic speech technology has made social robots, which use voice interfaces for interaction, become mainstream. From the perspective of the listener, the language communication cues belong to two sources of information, one is verbal channel, and the other is vocal channel. To improve the robot's voice interaction experience, the adjustment of verbal and vocal channels are crucial. But the current empirical research on the impact of social robots' voice on users' experience is very limited, and it is impossible to establish a set of application guidelines. 
With the continuous improvement of robot-related technologies such as artificial intelligence, in the future, social robots in different industries should be able to automatically adjust their own voice parameter settings (such as fundamental frequency and speech rate) according to the changes in their dialogue content, potentially improving their own dialogue. The degree of humanization, getting rid of the rigid and mechanical image, allows artificial intelligence to play its greatest value in social robot applications. Therefore, the purpose of this research is to improve the humanization degree which is shown in the voice interaction of social robots used for education and to increase user acceptance. Specifically, this study explores the effects of social robot dialogue types and voice parameters (gender, fundamental frequency, speech rate) used in education on their perceived personality traits and acceptance, and compares the differences and preferences of users' judgments of personality traits triggered by the voice of educational robots.

\section{The Voice Research of Robots \\ 2.1. Sound and Vocal Characteristics}

As we all know, sound is caused by vocal cords or vocal vibrations, which causes air molecules to vibrate to form sound waves. There are three key factors of sound: pitch, volume, and timbre. Among them, (1) tone refers to the frequency of sound wave vibration, the unit is hertz $(\mathrm{Hz})$, the faster the vibration, the higher the frequency, and the higher the sound frequency.(2) Volume refers to the amplitude of sound wave, the unit is decibel (dB), the greater the amplitude of sound wave vibration, the greater the volume.(3) Tone refers to the waveform of the sound wave itself. The sound waves, which are generated by the vibration of different sounding bodies, are different.Human voice is more complicated than sound, because human voice comes from the vibration of vocal cords to drive air to resonate in the chest cavity, abdominal cavity and skull, and then it will be affected by the shape of the nose and lips during the output process Interference[9].Previous researchers, on the one hand, asked the vocalist to pronounce normally when receiving vocal research samples, on the other hand, they used Fundamental frequency (F0), also known as fundamental frequency, to describe the vocal frequency[10].The fundamental frequency refers to the lowest frequency of the composite wave formed by human voice in vocalization, which can be measured by using spectrum analysis software[11][12]. It is the current voice that is the most commonly used by research indicators in the study of acoustics.

\subsection{The Voice Research of Robots}

Regardless of whether it is a human or a robot, some characteristics of speech, such as fundamental frequency (FO), loudness and speech rate are considered as the basic indicators of speech showing different personality traits [13]. The loudness, speech rate, frequency, and pause of the voice can help people understand the content and emotional state of the other party [14].

The control of Fundamental Frequency is the most commonly used variable for generating different speech [15][16][17], which can be used for Control the gender, personality traits of the voice. For example, [4] used fundamental frequency control to generate robots with male and female voices. [18] controlled the fundamental frequency in the study to produce robots with extroverted and introverted personality traits. The pitch range refers to the difference between the highest frequency and the lowest frequency in a piece of speech[19]. The frequency range affects the listener's emotions, usually the frequency range of sadness is smaller, and the frequency range of happiness or anger is larger. Loudness is usually measured in decibels $(\mathrm{dB})$, which is a logarithmic unit of measurement of the ratio of two values [19]. A soft voice (low volume) indicates boredom, while a loud voice indicates emotions such as happiness or anger. Speech rate refers to the speed of speech and pauses in sentences. Speech rate is usually measured in words/minute (wpm: word-per-minute), or syllables/minute (spm: syllables-per-minute ), the pause in the statement can be measured in seconds. Speech rate can affect human emotions and perceived personality traits of robots. Happiness and anger usually speak faster, and sadness usually speak slower[20]. Harwood [21]research believes that when the speed of English is 125-225 words/minute, it will not have a significant impact on the understanding of native English speakers. Jones et al.[22]defined a normal speech rate of 155 words per minute, or 237 syllables per minute.

In addition to the above factors, Gender is also a key factor. Studies have shown that human voice has obvious dimorphism, which is due to differences in the physiological structure of the vocal cords between men and women[23].The 
length of the male vocal tract is longer than that of the female, so the overall frequency of the sound is lower. People have different preferences for voice gender, and this preference is related to gender stereotypes.

\section{Research on the Perceived Personality Traits of Social Robots}

Among the various of social characteristics, personality traits are considered to be important factors in interpersonal relationships and human-computer interaction. According to the CASA paradigm "Computer as Social Actors", people tend to think of computers and robots as a social role[24].People will be willing to apply human social norms to computers and robots, treating them as if they were humans. The previous research in the field of psychology has provided a sufficient theoretical based on human personality traits[25]. One of the most widely accepted in robotics research is the Big Five Theory[26] which has been developed in the field of psychology for more than 50 years [25]. Norman [26] proposed five personality dimensions (emotional stability, extroversion, pleasantness, rigor, culturality) in the study, which is the basis of the later five personality.Subsequently, Costa et al.[27]others further defined and named the five major personalities as Extraversion, Agreeableness, Conscientiousness, Neuroticism, Openness experience, and the published Five Factor Scale (NEO-PI-R) has developed into the most perfect five personality models[27][28].

\section{Methods}

\subsection{Voice Sample Setting and Production}

There are generally two ways to make sounds used in robot experiments. The first is to record human voices, and the second is to use synthetic voices. The advantage of recording human voice is that it is rich in emotions, but it is difficult to quantify and it is difficult to copy. Although the synthesized vocals are not as rich in emotion as in recorded vocals. They are easy to copy and help subsequent verification.Chinese Speech System HKUST Xunfei Synthetic Speech is one of the most powerful systems for synthesizing Chinese speech. Its synthesized speech is closer to the real human voice than the commonly used Chinese synthesized speech systems such as Miss Google and Mac computer speech. Therefore, the robot sound of this research was selected to be produced by the HKUST Xunfei speech synthesis system.

The previous research results of this study show that the sound samples of medium and low fundamental frequencies are highly accepted in the application of educational robots, and there is obviously a dimorphism of human voice.Therefore, this study selected two adult male and female voices generated by the HKUST Xunfei speech synthesis system. The female voice comes from the human voice: Xiao Yan, and the male voice comes from the human voice: Yifeng. Tay et al.[4] defined fast speech rate $=216$ words per minute and slow speech rate $=184$ words per minute in a previous study. Dou et al. [12] defined Female frequency $=228.49 \mathrm{~Hz}$; male frequency $=133.55 \mathrm{~Hz}$. Based on the above research, this study uses Adobe Audition CC to control the fundamental frequency and speech rate[29]. Based on the original voice generated by the system, the tone changes, decreases, accelerates, and decelerates to control the type of voice change. Because Apple et al.[30] explored the effect of vocal frequency and speech rate on the listener's perception of the speaker's personal attribute, the experiment used $80 \%-120 \%$ as the frequency change interval. The speed is $70 \%-$ $130 \%$ as the change interval. High frequency voice is used $20 \%$. In order for listeners to be able to recognize the difference in the frequency of two voices, the fundamental frequency of the sound needs to differ by more than 50[31]. After generating samples of speech parameters, we used praat to analyze the average speech rate/average fundamental frequency of the speech samples.

\subsection{Robot Dialogue Content Setting}

Previous research usually divided the robot dialogues system into two types, according to the different contents of the dialogues, task-oriented dialogues and non-task-dialogues.The task-oriented dialogues of the robots, applied in the education industry in this study, refers to the dialogue process in the study of Serholt [32], and the dialogue on the theme of the temple door stone lion. Non-task-dialogues scripts have no conversation theme and are mainly based on chat.The research collected the views of the subjects $(\mathrm{N}=5$, male $=2$, female $=3$, mean age $=20.40$, standard deviation $=0.80)$ on the non-task-dialogues of educational robots. Invite them to provide five sets of questions based on "what do you want to talk about if you chat with an educational robot, and how to answer them", and choose five sets of dialogues. 


\subsection{Robot Personality Trait Questionnaire}

The experiment used a questionnaire to collect data, and the dependent variable was the evaluation of social robot personality traits. Discussed earlier on the personality traits of robots. Most of the current researches use the Big Five Theory as the theoretical basis, but it is difficult for the subjects to complete a lengthy questionnaire in the robot experiment[33], and the first-person question questioning method is not suitable for robot experiments.[34] In the previous study, 13 vocabularies from the five major personality theories were used to investigate the impact of the robot's shape on the special personality perception. The advantage of this questionnaire is to improve the problems of the five personality scale question items and the first-person questioning method. It has been used in the measured robot's perceptual personality quality, so this research use this questionnaire item to measure. In addition, this study also add an overall evaluation question based on this questionnaire.Questions were measured by using the 7-point Likert scale, $1=$ very disagree, $7=$ very agree

\subsection{Experimental Design}

The humanoid robot Alpha 1 Pro (Figure 1) was selected for experiments. AlphaIPro is a small robot (401*198*124mm) developed by UBtech, which has been used in previous robot research[35].Alpha 1 Pro has a built-in 3w speaker, but Alpha 1 Pro cannot produce many types of sound. In order to facilitate the experiment, a small Bluetooth speaker was connected to the robot and behind the robot. Based on the principles of VHRI's experimental method, the robot Alpha 1 Pro communication with a real person, and recorded the video of educational robot, obtaining 16 video clips. In order to break the limitations of technology and improve the prospectiveness of this research, the main experimental method used in this study is video-based human robot interaction (VHRI), which is one of the mainstream experimental examples of social robot research[36][37]. The subjects can start the experiment by watching the recorded video of the actual interaction between the person and the robot[38], which can try to avoid the change in feelings caused by the change in the dialogue between the individual and the robot.

There are total of 43 subjects tested. After excluding invalid questionnaires, 32 valid questionnaires were finally obtained. (mean age $=21.63$, standard deviation=2.13, age range 19-29, male=15, female $=17$ ) The subjects from a college of design in Taiwan and a business school in a college in mainland China. Before the experiment, each participant who participates in the experiment will be required to make an appointment in an experimental group 'each group of 1-3 subjects, plus one staff member. When the subjects arrive at the laboratory, they will get an information sheet to introduce the experiment content, telling them that they only need to pay attention to the robot's voice and dialogues content during the experiment. And then they need to complete a simple questionnaire to collect basic demographic information, such as their age, gender, and experience by using robots. In the experiment, the distance between the subjects and the video is within 2 meters, and the staff will play 16 experimental videos in random order. After each video is played, the staff will tell the subject to start filling out the questionnaire and confirm that everyone has completed the questionnaire after playing the next video. At the end of the experiment, each subject will receive 16 questionnaires.

\section{Result}

\subsection{Reliability Analysis of Questionnaire of Personality Trait of Robot Perception}

Data was analyzed using SPSS 21. The five personality traits showed that Cronbach's Alpha were all higher than 0.60. The results showed that the reliability of the five facets was between 0.6-0.8, and the reliability was higher.

\subsection{Education industry, the type of dialogues and speech parameters on perceptual personality traits}

For educational robots, adjusting the dialogue types and fundamental frequency factor can significantly improve the robot extroversion of user's perception. Therefore, when educational robots need to highlight extroversion, non-task dialogue can be used combination with high fundamental frequency sound settings. On the characteristics of agreeable (A), it can be found that for educational robots, adjusting the voice gender, fundamental frequency, and speech rate factor can significantly improve the user's perception of robots' agreeable. Therefore, when educational robots need to highlight the agreeable, nontask dialogues can be used and combined with male voices low frequency and fast voice settings. On the characteristics of conscientious (C) when the educational robot needs to highlight the conscientious, male voices, low frequency, fast speech speed settings can be used. About the anti-neurotic $(\mathrm{N})$ that for educational robots, adjusting the dialogue fundamental 
frequency and speech rate factor can significantly improve the robot's anti-neurotic of user's perception. Therefore, when educational robots need to highlight the Anti-neurotic, male voices combined with low frequency, fast speech speed setting can be used. Openness $(\mathrm{O})$ When the educational robot needs to highlight the openness, it can adopt a task-oriented dialogue with female voices, low frequency and slow speech voice settings.

\section{Discussion}

\subsection{Future Suggestions}

In addition to the education industry mentioned in this research, the application prospects of social robots in certain occupational fields are also very broad, such as shopping mall robot receptionists, public assistants, family companion robots, etc.[39]-[44]. In the future, we can refer to the research on the setting of educational robot voice parameters and dialogue types, and continue to study for different industries.

\subsection{Limitations}

In the HRI experiment, the sound and shape of the robot will inevitably be linked together, and it is almost impossible to use a neutral humanoid robot without gender and age implications. For example, the commonly used robot "Pepper" is thought to look like a teenage boy. Therefore, we emphasized to the subjects in the experiment that they only evaluate the feeling caused by the robot sound, ignoring the shape part, hoping that this can offset the impact of some shapes. Subjects in the experiment are easily influenced by the content of the dialogue. Although this study considers the influence of dialogues content, the dialogues content is divided into two types: task-oriented and non-task-oriented, however, there is still a big adjustment space in the details of the specific dialogues. Each sentence in the interaction may affect the subject's judgment of the robot's personality traits. In future research, we can consider modifying the content of the dialogues, and further explore the impact of the change in the dialogues content on the personality traits of robot perception.

\section{Conclusion}

This study provides guidelines for Voice Parameters of Social Robots Applied in Education Industry.Through experiments, it is found that the voice of the educational robot can be male or female. But the difference is that male voices low frequency and slow voice setting are more receptive by the users when conducting non-task dialogues. In task-oriented dialogues, the female voices low frequency and slow voice parameters are more accepted by the users. Therefore, it is best for educational robots to use low frequency and slow voice parameter settings. When highlighting the five personality traits of educational robots, it is best to set the low frequency and fast voice of male voices under non-task dialogues. In taskoriented dialogues, in addition to highlight extroversion personality traits need to set high frequency voices, low frequency voices are recommended for the other four personality traits. This research, through the design guidance of speech parameters, will help educational robots have a higher sense of user acceptance and a better user experience in the education field in the future, and also provide speech parameters for social robots in other fields. The design provides design reference.

\section{Acknowledgements}

The study was financially supported by Ministry of Science and technology, Taiwan (grant number MOST 107-2221E-036-014-MY3) and Tatung Company, Taiwan (grant number E10603-P01-037). 


\section{References}

[1] T. Belpaeme, J.Kennedy,A.Ramachandran,B.Scassellati and F. Tanaka, "Social robots for education: A review,"Science Robotics, 21(3), eaat5954, 2018.

[2] J.Martmez-Miranda, H.Perez-Espinosa, I. Espinosa-Curiel, I. Avila-George and J. Rodriguez-Jacobo, “Age-based differences in preferences and affective reactions towards a robot's personality during interaction," Computers in Human Behavior,2018.

[3] S. J. Stroessner and J.Benitez, "The Social Perception of Humanoid and Non-Humanoid Robots: Effects of Gendered and Machinelike Features," International Journal of Social Robotics, 77, 305-315,2019.

[4] B. Tay, Y.Jung and T. Park, "When stereotypes meet robots: the double-edge sword of robot gender and personality in human-robot interaction," Computers in Human Behavior, 38, 75-84,2014.

[5] Walters. (2008). The design space for robot appearance and behaviour for social robot companions. (Ph.D). University of Hertfordshire, Hertfordshire, UK. Available: https://uhra.herts.ac.uk/handle/2299/1806

[6] Walters, D. Syrdal, K.Koay, K.Dautenhahn and R.Te Boekhorst, Human approach distances to a mechanical-looking robot with different robot voice styles. Proceedings of the 17th IEEE International Symposium on Robot and Human Interactive Communication, RO-MAN,2008.

[7] T. Fong, I. Nourbakhsh and K.Dautenhahn, A survey of socially interactive robots. Robotics and Autonomous Systems, 2003.

[8] N. Mavridis, "A review of verbal and non-verbal human-robot interactive communication.,"Robotics and Autonomous Systems, 63, 22-35,2015.

[9] M. Latinus and P. Belin"Human voice perception,"Current Biology, 27(4), R143-R145,2011.

[10] C. R.Hodges-Simeon, S. J. Gaulin and D. A. Puts, "Different vocal parameters predict perceptions of dominance and attractiveness," Human Nature, 27(4), 406-427,2010.

[11] J. Crumpton and C. L. Bethel, "A survey of using vocal prosody to convey emotion in robot speech," International Journal of Social Robotics, 5(2), 271-285, 2016.

[12] X. Dou, C.-F. Wu, K.-C. Lin and T.-M. Tseng, "The Effects of Robot Voice and Gesture Types on the Perceived Robot Personalities," International Conference on Human-Computer Interaction, Orlando, Florida, USA,2019.

[13] C. Nass and K. M. Lee, "Does computer-generated speech manifest personality? an experimental test of similarityattraction," in proceedings of the SIGCHI conference on Human factors in computing systems - CHI '00, 2, 329336,2000a.

[14] M. Morningstar, M. A.Dirks and S. Huang, "Vocal Cues Underlying Youth and Adult Portrayals of Socio-emotional Expressions," Journal of Nonverbal Behavior, 2017.

[15] B. L. Brown, W. J. Strong, and A. C. Rencher, "Perceptions of personality from speech: Effects of manipulations of acoustical parameters," The Journal of the Acoustical Society of America, 54(1), 29-35, 1973.

[16] W. Gu, T. Zhang and H.Fujisaki, Prosodic analysis and perception of Mandarin utterances conveying attitudes. The Twelfth Annual Conference of the International Speech Communication Association,2011.

[17] J. A. Sereno, H.Lee and A.Jongman, "Effects of speaking rate and context on the production of Mandarin tone," Paper presented at the ICPhS,2015.

[18] R. C. S. Chang, H. P.Lu and P. Yang, "Stereotypes or golden rules? Exploring likable voice traits of social robots as active aging companions for tech-savvy baby boomers in Taiwan," Computers in Human Behavior, 84, 194-210, 2018.

[19] J. Crumpton andC. L.Bethel, "A survey of using vocal prosody to convey emotion in robot speech," International Journal of Social Robotics, 5(2), 271-285, 2016.

[20] G. L.Huttar, "Relations between prosodic variables and emotions in normal American English utterances," Journal of Speech and Hearing Research, 77(3), 481-487,1968.

[21] K. A. Harwood, III. Listenability and rate of presentation. Communications Monographs, 22(1), 57-59,1955.

[22] C.Jones, L.Berry and C. "Stevens, Synthesized speech intelligibility and persuasion: Speech rate and non-native listeners," Computer Speech \& Language, 27(4), 641-651,2007. 
[23] X. Liu and Y. Xu, What makes a female voice attractive? The 17th International Congress of Phonetic Sciences (ICPhS), Hong Kong, China, 2011.

[24] C. Nass, J. Steuerand E. R. Tauber, Computers are social actors. Conference companion on Human factors in computing systems - CHI '94,1994.

[25] L. R. Goldberg, "An alternative 'description of personality' : the big-five factor structure," Journal of Personality and Social Psychology, 59(6), 1216,1990.

[26] W. T.Norman, "Toward an adequate taxonomy of personality attributes: Replicated factor structure in peer nomination personality ratings," The Journal of Abnormal and Social Psychology, 66(6), 574,1963.

[27] P. T. Costa and R. R. McCrea, Revised neo personality inventory (neo pi-r) and neo five-factor inventory (neoffi):Psychological Assessment Resources, 1992.

[28] P. T. Costa and R. R. McCrea, The Revised NEO Personality Inventory (NEO-PI-R): Sage Publications, Inc,2008.

[29] C. R.Pernet and P. Belin, "The role of pitch and timbre in voice gender categorization.,"Frontiers in psychology, 3, 23,2012.

[30] W. Apple, L. A. Streeter and R. M. Krauss, "Effects of pitch and speech rate on personal attributions," Journal of Personality and Social Psychology,1979.

[31] A. Niculescu, B. van Dijk, B. Nijholt, H. Li and S. L. See, "Making social robots more attractive: the effects of voice pitch, humor and empathy," International Journal of Social Robotics, 5(2), 171-191,2013.

[32] 32.S. Serholt, "Breakdowns in children's interactions with a robotic tutor: A longitudinal study.," Computers in Human Behavior, 81, 250-264,2018.

[33] R. W.Robins, J. L.Tracy, K.Trzesniewski, J. Potter and S. D. J. J. o. r. i. p. Gosling, (2001). Personality correlates of self-esteem. i5(4), 463-482,2001.

[34] J.Hwang, T.Park and W.Hwang, "The effects of overall robot shape on the emotions invoked in users and the perceived personalities of robot," Applied Ergonomics, 44(3), 459-471,2013.

[35] J. Li, H.Peng,H. Hu, Z. Luo and C. Tang, "Multimodal Information Fusion for Automatic Aesthetics Evaluation of Robotic Dance Poses," International Journal of Social Robotics, 1-16,2018.

[36] K.Dautenhahn, "Methodology \& themes of human-robot interaction: A growing research field," International Journal of Advanced Robotic Systems, 4(1), 15,2017.

[37] R. van den Brule, R. Dotsch, G.Bijlstra, D. H. J.Wigboldus and P. Haselager, "Do Robot Performance and Behavioral Style affect Human Trust?: A Multi-Method Approach,” International Journal of Social Robotics, 2014.

[38] L.Takayama, D.Dooley and W. Ju, Expressing Thought: Improving Robot Readability with Animation Principles, 2011.

[39] H. Admoni, and B. Scassellati, "Roles of robots in socially assistive applications," Paper presented at the IROS 2014 Workshop on Rehabilitation and Assistive Robotics, Chicago, U.S, 2014.

[40] F. Bertacchini, E. Bilotta and P. Pantano, Shopping with a robotic companion. Computers in Human Behavior, 77, 382-395, 2017.

[41] E. Broadbent, D. A. Feerst, S. H. Lee, H. Robinson, J. Albo-Canals, H. S. Ahn and B. A. MacDonald, "How could companion robots be useful in rural schools?" International Journal of Social Robotics, 10(3), 295-307, 2018.

[42] C.Kim, D.Kim, J.Yuan, R. B.Hill, P. Doshi and C. N. Thai, "Robotics to promote elementary education pre-service teachers' STEM engagement, learning, and teaching," Computers \& Education, 91, 14-31,2015.

[43] H.Robinson, B.MacDonald and E. Broadbent, "The role of healthcare robots for older people at home: A review," International Journal of Social Robotics, 6(4), 575-591,2014.

[44] D. S.Syrdal,K. L. Koay,M. L. Walters and K. Dautenhahn, A personalized robot companion? - The role of individual differences on spatial preferences in HRI scenarios. In proceedings IEEE International Workshop on Robot and Human Interactive Communication,2007. 